Research Paper

\title{
Sleep Duration and Patterns in Chinese Older Adults: a Comprehensive Meta-analysis
}

\author{
$\mathrm{Li} \mathrm{Lu}^{1 *}$, Shi-Bin Wang ${ }^{1,2 *}$, Wen-Wang Rao ${ }^{3 *}$, Gabor S. Ungvari ${ }^{4,5}$, Chee H. Ng ${ }^{6}$, Helen F.K. Chiu ${ }^{7}$, Juan \\ Zhang $^{8}$, Changgui Kou ${ }^{3}$, Fu-Jun Jia ${ }^{2 凶}$, Yu-Tao Xiang ${ }^{\circledR}$ \\ 1. Unit of Psychiatry, Faculty of Health Sciences, University of Macau, Macao SAR, China; \\ 2. Guangdong Mental Health Center, Guangdong General Hospital \& Guangdong Academy of Medical Sciences, Guangzhou, Guangdong, China; \\ 3. Department of Epidemiology and Biostatistics, School of Public Health, Jilin University, Changchun, China; \\ 4. University of Notre Dame Australia / Marian Centre, Perth, Australia; \\ 5. School of Psychiatry \& Clinical Neurosciences, University of Western Australia, Perth, Australia; \\ 6. Department of Psychiatry, University of Melbourne, Melbourne, Victoria, Australia; \\ 7. Department of Psychiatry, Chinese University of Hong Kong, Hong Kong SAR, China; \\ 8. Faculty of Education, University of Macau, Macao SAR, China. \\ * These authors contributed equally to the work.
}

$\triangle$ Corresponding authors: Dr. Yu-Tao Xiang, 3/F, Building E12, Faculty of Health Sciences, University of Macau, Avenida da Universidade, Taipa, Macau SAR, China. Fax: +853-2288-2314; Phone: +853-8822-4223; E-mail: ytxiang@umac.mo; or Dr. Fu-Jun Jia, Guang Dong Mental Health Centre, Guangdong province, China. E-mail: jiafujun@126.com

(c) Ivyspring International Publisher. This is an open access article distributed under the terms of the Creative Commons Attribution (CC BY-NC) license (https://creativecommons.org/licenses/by-nc/4.0/). See http://ivyspring.com/terms for full terms and conditions.

Received: 2017.02.16; Accepted: 2017.04.11; Published: 2017.05.16

\begin{abstract}
This meta-analysis examined the mean sleep duration and patterns in Chinese older adult population. A literature search was systematically conducted covering major English (PubMed, Embase and PsycINFO) and Chinese (Chinese National Knowledge Infrastructure (CNKI), WanFang and SinoMed) databases. Data in studies with the mean and standard deviation of sleep duration and/or the proportion of short and long sleep durations in Chinese older adults were extracted and pooled using random-effects models. Subgroup analyses were conducted according to gender, region, area, survey time and sample size. A total of 36 studies with 150,616 subjects were included for analyses. The pooled mean sleep duration of 21 studies with available data was 6.82 hours/day (95\% Cl: 6.59-7.05 hours/day). The estimated proportions of sleep duration $<5$ hours/day, $<6$ hours/day, $<7$ hours/day were $18.8 \%$ (95\% Cl: $1.7 \%-35.9 \%), 26.7 \%(95 \% \mathrm{Cl}$ : $19.7 \%-33.7 \%)$ and $42.3 \%$ (95\% Cl: 34.8\%-49.8\%), respectively. The pooled proportions for long sleepers were $22.6 \%$ (95\% Cl: 13.9\%-31.4\%) (>8 hours/day) and $17.6 \%$ (95\% Cl: $12.4 \%-22.9 \%)(>9$ hours/day). Given the adverse effects of unhealthy sleep patterns, health professionals should pay more attention to sleep patterns in this population in China.
\end{abstract}

Key words: Sleep duration, old adult, meta-analysis, China.

\section{Introduction}

Sleep pattern is closely associated with health and well-being. Emergent evidence indicates that both insufficient and excessive sleep durations are linked with poor physical and mental health [1-6]. In addition, a U-shaped relationship between sleep duration and mortality was found in many studies [7, 8]. Understanding the distribution of sleep duration and sleep patterns in a population is essential for health professionals to examine the sleep-related health problems and implement effective measures to improve unhealthy sleep habits.

Population aging has been a growing health challenge worldwide, especially in developing countries, such as China $[9,10]$. The Chinese Ministry of Civil Affairs reported that the number of elderly persons aged 60 years and above have reached 222 million in China, accounting for $16.1 \%$ of the whole population at the end of 2015 [11]. Sleep problems 
including short and long sleep durations have been common in older adult population globally [1-6, 12]. In China, sleep patterns have been found to vary greatly across different studies [13-15]. One major reason is lack of gold standard criterion of short and long sleep duration. The recent recommendation made by the National Sleep Foundation suggested that different sleep durations are appropriate for different age groups [16]. Older people aged 65 years and above were recommended to sleep 7-8 hours per day; less than 5 or more than 9 hours per day are not recommended [16].

To the best of our knowledge, there have been no meta-analysis or systemic review of the sleep duration and patterns among older adults in China. The objective of this study was to summarize the data from observational studies and then estimate the mean sleep duration and proportion of short and long sleepers in Chinese older adult population.

\section{Methods}

\section{Search strategy}

This meta-analysis was performed based on the Preferred Reporting Items for Systematic Reviews and Meta-Analyses (PRISMA) statement. Two reviewers (LL and RWW) identified studies independently from PubMed, Embase, PsycINFO, Chinese National Knowledge Infrastructure(CNKI), WanFang, SinoMed from inception until September 10, 2016, using the search terms as follows: (sleep time OR sleep duration OR sleep habit OR sleep pattern OR insomnia OR sleep problem OR sleep disturbance OR sleep disorder OR sleep quality OR sleep symptom) AND (epidemiology OR cross-sectional study OR prevalence OR rate) AND (older adults OR elderly OR aged OR aging) AND (China OR Chinese). Additional studies were searched manually from the references of the selected publications.

\section{Study selection}

Studies that met the following criteria were included: a) cross-sectional or cohort studies conducted in China; b) sleep duration expressed as mean and standard deviation (SD) or as the proportion of short ( $<5$ hours/day, $<6$ hours/day, or $<7$ hours/day) and long ( $>8$ hours/day or $>9$ hours/day) sleep duration in adults aged $\geq 60$ years according to the recommendation by the Chinese Ministry of Health; c) sample size $\geq 100$; d) availability in full text in Chinese or English. For cohort studies, only the baseline data were extracted for analyses.

Studies on special populations (e.g., army, retired people, empty nesters, people with major medical conditions) or specific settings (e.g., hospitals or nursing homes) and those using convenience sampling or without details on sampling process were excluded.

Two reviewers (LL and RWW) screened titles and abstracts of the initial search results independently. If there were more than one article based on the same study, only the one with the largest sample size and complete information was included for analysis.

\section{Data extraction and quality assessment}

Two reviewers (LL and RWW) conducted the data extraction independently. Disagreements emerged in the procedures were resolved by a discussion with a third reviewer (WSB). The following information was extracted and tabulated: study setting, sampling method, sample size, characteristics of the participants, and sleep duration with quantitative data. If there was more than one arm using different cut-offs of sleep duration in one study, then these arms were analyzed separately. The quality of included studies was assessed using the 22-item Strengthening the Reporting of Observational Studies in Epidemiology (STROBE) [17]. In this meta-analysis, studies with a score of $>11$ were rated as "good quality" [18].

\section{Statistical analysis}

The pooled mean of sleep duration, proportion of short and long sleep durations, and their 95\% confidence intervals (CI) were calculated using random-effects models. The bedtime, sleep latency, time to go to bed and to get up were also pooled. Heterogeneity between studies was measured by $I^{2}$ statistic; $I^{2}>50 \%$ was considered as high heterogeneity [19]. Subgroup analyses were performed to explore possible sources of heterogeneity according to age groups $(60 \sim / 70 \sim / \geq 80$ years old; for sleep duration only), gender, area (rural/urban), region (east, central and west parts of China according to the Chinese economic zone division), sample size and survey time (dichotomized using median splitting method). If there were more than 10 studies, meta-regression models were used to detect heterogeneity for continuous variables [20]. Funnel plots, Begg's test [21] and Egger's regression model [22] were used to evaluate publication bias. Sensitivity analysis was carried out by removing each study individually to evaluate the consistency of the results. Comprehensive Meta-Analysis software version 2 (Biostat Inc., Englewood, New Jersey, USA) was used to perform the subgroup analyses and STATA version 12.0 (Stata Corporation, College Station, Texas, USA) was used to conduct other analyses. Significance level was set at 0.05 (two-tailed). 


\section{Results}

\section{Studies selection results and basic characteristics}

A total of 6,598 potential papers were identified, of which 36 papers published in English $(n=10)$ and Chinese $(n=26)$ with 150,616 subjects met the selection criteria and were included for analyses (Figure 1). A total of 21 studies reported mean and SD of sleep duration, 22 studies reported the proportion of short sleepers (15 studies reported the data of sleeping $<6$ hours/day and 11 studies reported the data of sleep $<7$ hours/day) and 17 studies reported the proportion of long sleepers (10 studies reported the data of sleeping $>8$ hours/day and 9 studies reported the data of sleep $>9$ hours/day). One study provided data of Han and Korean Chinese separately, therefore we extracted and analyzed the data as two arms [23]. Comprehensive characteristics of the eligible studies are shown in Table 1.

\section{Quality assessment and publication bias}

The mean STROBE score of the studies was 18, ranging from 14 to 21. All studies were classified as good quality. Supplemental Figure 1 shows the funnel plot of the 21 studies with mean and SD of sleep duration, the Begg's $(Z=-1.15, P=0.264)$ and Egger's tests $(t=-0.44,95 \% \mathrm{CI}:-19.1-12.5, P=0.667)$ did not reveal any publication bias. Similarly, no publication bias was found in other meta-analyses (Table 2).

\section{Sleep duration of Chinese elderly}

The meta-analysis revealed that the mean sleep duration was 6.82 hours/day (95\% CI: 6.59-7.05 hours/day) (Figure 2). The proportion of short and long sleepers are presented in Table 2 . The proportion of short sleepers was $18.8 \%$ (95\% CI: $1.7 \%-35.9 \%$ ), $26.7 \%$ (95\% CI: $19.7 \%-33.7 \%$ ) and $42.3 \%$ (95\% CI: $34.8 \%-49.8 \%$ ), when defined as less than 5 hours/day, 6 hours/day and 7 hours/day, respectively. In contrast, the proportion of long sleepers was $22.6 \%$ $(95 \%$ CI: $13.9 \%-31.4 \%)$ and $17.6 \% \quad(95 \%$ CI: $12.4 \%-22.9 \%$ ), when defined as more than 8 hours/day and 9 hours/day, respectively.

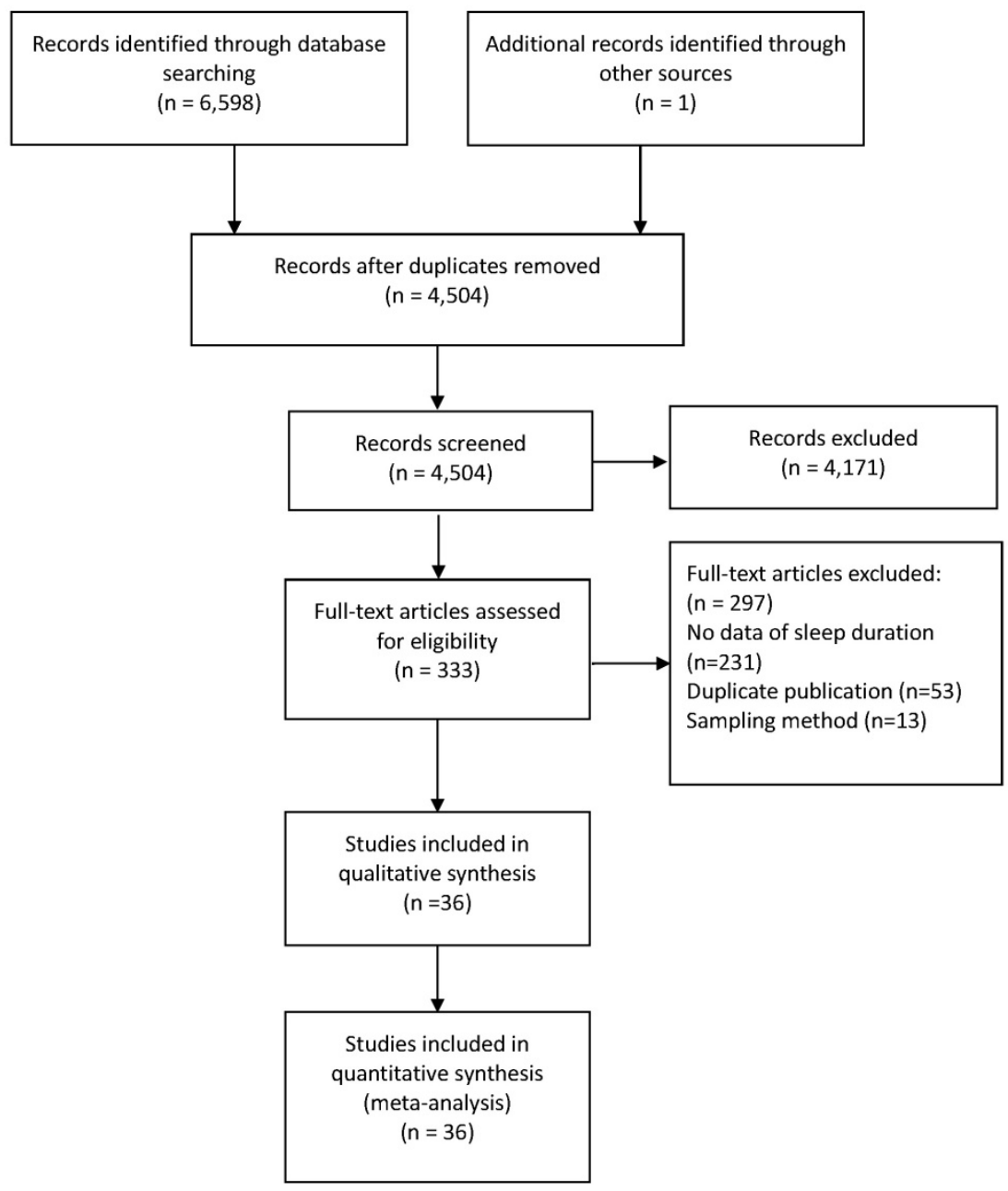

Figure 1. Flowchart of the selection of studies 
Table 1. Characteristics of included studies

\begin{tabular}{|c|c|c|c|c|c|c|c|c|c|c|c|c|}
\hline \multirow[t]{2}{*}{ No. } & \multirow{2}{*}{$\begin{array}{l}\text { Author } \\
\text { (Publication year, } \\
\text { language) }\end{array}$} & \multirow[t]{2}{*}{$\begin{array}{l}\text { Sampling } \\
\text { method }\end{array}$} & \multirow{2}{*}{$\begin{array}{l}\text { Survey } \\
\text { time } \\
\text { (year) }\end{array}$} & \multirow[t]{2}{*}{$\begin{array}{l}\text { Sample } \\
\text { size }\end{array}$} & \multicolumn{2}{|c|}{$\begin{array}{l}\text { Age } \\
\text { (years) }\end{array}$} & \multirow[t]{2}{*}{$\begin{array}{l}\text { Urban/ } \\
\text { Rural }\end{array}$} & \multirow[t]{2}{*}{$\begin{array}{l}\text { Female } \\
(\%)\end{array}$} & \multirow[t]{2}{*}{ City/Province } & \multirow[t]{2}{*}{ Area } & \multirow[t]{2}{*}{ Sleep information } & \multirow[t]{2}{*}{$\begin{array}{l}\text { STROBE } \\
\text { score }\end{array}$} \\
\hline & & & & & Mean & SD & & & & & & \\
\hline 1 & Ding X.J. $(1997$, C) & S, C, R & 1992 & 2779 & NR & NR & Mixed & NR & Beijing & East & $\mathrm{M},<6,>9$ & 14 \\
\hline 2 & $\begin{array}{l}\text { Chiu H.F.K. et al. } \\
(1999, \text { E) }\end{array}$ & S, R & 1995 & 1034 & NR & NR & Urban & 51.26 & Hong Kong & East & $\mathrm{M}, \mathrm{SD}$ & 21 \\
\hline 3 & $\begin{array}{l}\text { Liu L.Q. et al. (2001, } \\
\text { C) }\end{array}$ & S, C, R & 1997 & 1805 & 70.62 & 5.27 & Urban & 52.47 & $\begin{array}{l}\text { Jinan,Wendeng,Liaocheng, } \\
\text { Zaozhuang,Binzhou/Shandong }\end{array}$ & East & M, SD, TB, SL, TG & 20 \\
\hline 4 & $\begin{array}{l}\text { Lin D.Y. et al. (Han) } \\
(2005, C)\end{array}$ & $\mathrm{R}$ & 2004 & 469 & 67.7 & 6.0 & Urban & 54.37 & Yanji/Jilin & Middle & M, SD, TB, TG, BT & 16 \\
\hline 5 & $\begin{array}{l}\text { Lin D.Y.et al. } \\
\text { (Korean) }(2005, C)\end{array}$ & $\mathrm{R}$ & 2004 & 494 & 68.5 & 6.2 & Urban & 67.00 & Yanji/Jilin & Middle & M, SD, TB, TG, BT & 16 \\
\hline 6 & $\begin{array}{l}\text { Zhang Q.H. et al. } \\
(2006, C)\end{array}$ & M, R & 2004 & 25061 & 71.2 & 9.5 & Urban & NR & Beijing & East & $\begin{array}{l}\text { M, SD, }<6,>8, T B, \\
\text { SL, TG, BT }\end{array}$ & 15 \\
\hline 7 & $\begin{array}{l}\text { Lan T.Y. et al. (2007, } \\
\text { E) }\end{array}$ & M, PPS & 1993 & 3079 & 71.28 & 5.58 & NR & 43.23 & Taiwan & East & $<7,>8,>9$ & 19 \\
\hline 8 & Liu Y. $(2007$, C) & $\mathrm{C}, \mathrm{R}$ & 2005 & 2304 & 74.5 & 7.7 & Urban & 57.94 & Shanghai & East & $\mathrm{M}, \mathrm{SD}, \mathrm{TB}, \mathrm{SL}$ & 20 \\
\hline 9 & $\begin{array}{l}\text { Yao K.W. et al. } \\
(2008, \mathrm{E})\end{array}$ & M, R & NR & 187 & 72.13 & 4.93 & Urban & 48.66 & Taipei & East & $\mathrm{M}, \mathrm{SD}, \mathrm{SL}, \mathrm{BT}$ & 20 \\
\hline 10 & Liu A.L. $(2008, C)$ & $\mathrm{R}, \mathrm{C}$ & NR & 5390 & NR & NR & NR & 51.54 & Zhengzhou,Kaifeng/Henan & Middle & $<6,>9$ & 18 \\
\hline 11 & $\begin{array}{l}\text { Xiang Y.T. et al. } \\
(2009, \text { E) }\end{array}$ & S, M, PPS & NR & 1141 & NR & NR & NR & NR & Beijing & East & $<7,>8$ & 20 \\
\hline 12 & $\begin{array}{l}\text { Liu H.L. et al. (2009, } \\
\text { C) }\end{array}$ & $\begin{array}{l}\text { S, C, R, } \\
\text { PPS }\end{array}$ & 2006 & 664 & NR & NR & Urban & 53.92 & Shijiazhuang/Hebei & East & M, SD, TB, SL, TG & 18 \\
\hline 13 & Li J. $(2009$, C) & $\mathrm{R}$ & 2006 & 1006 & NR & NR & Mixed & NR & Tianjin & East & $\mathrm{M}, \mathrm{SD}$ & 17 \\
\hline 14 & Li H. et al. $(2009$, C) & $\mathrm{C}, \mathrm{R}$ & 2007 & 4237 & NR & NR & NR & 56.86 & Fuzhou/Fujian & East & $<6,>9$ & 15 \\
\hline 15 & Gu D. et al. $(2010, E)$ & $\mathrm{R}$ & 2005 & 15638 & NR & NR & Mixed & 57.23 & 22 provinces & & $\mathrm{M}, \mathrm{SD},<7,>9$ & 17 \\
\hline 16 & Li J. $(2010$, C) & S, C, R & 2009 & 1680 & 68.44 & 7.1 & Rural & 49.94 & Anhui & Middle & $\mathrm{M}, \mathrm{SD}, \mathrm{TB}, \mathrm{TG}$ & 20 \\
\hline 17 & Xie Z. et al. $(2010$, C) & S, C, R & 2009 & 1040 & 70.1 & 7.4 & Rural & 48.08 & Hengyang/Hunan & Middle & $\begin{array}{l}\text { M, SD, }<5,<6, \\
<7,>8,>9, \text { TB, TG }\end{array}$ & 19 \\
\hline 18 & $\begin{array}{l}\text { Wang W. et al. } \\
(2011, C)\end{array}$ & $\mathrm{R}, \mathrm{C}$ & NR & 145 & 63.91 & 2.94 & Urban & 55.17 & Beijing & East & $<6,>8$ & 17 \\
\hline 19 & He M.H. $(2011$, C) & $\mathrm{R}, \mathrm{C}$ & 2010-2011 & 1200 & 65.6 & 6.7 & Rural & 36.25 & Hong'an/Hubei & Middle & $\mathrm{M}, \mathrm{SD}$ & 15 \\
\hline 20 & Chen Z.Y. $(2011$, C) & $S, R$ & NR & 312 & 69.1 & NR & Rural & 58.65 & Western Hunan & Middle & $<6,>9$ & 17 \\
\hline 21 & $\begin{array}{l}\text { Wu C.Y. et al. }(2012, \\
\text { E) }\end{array}$ & $\mathrm{R}$ & 2001 & 100 & 74.7 & 5.3 & Urban & 55.00 & Taipei & East & $<5,<7$ & 20 \\
\hline 22 & $\begin{array}{l}\text { Wang Q. et al. (2012, } \\
\text { C) }\end{array}$ & $\mathrm{R}, \mathrm{S}, \mathrm{C}$ & 2011 & 404 & 71.18 & 6.61 & Urban & 63.12 & Xi'an/Shannxi & West & M, SD & 19 \\
\hline 23 & Li S.X. et al. $(2012, C)$ & $\mathrm{R}, \mathrm{C}$ & 2008-2009 & 753 & 67.9 & 7.05 & Urban & 51.13 & Tangshan/Hebei & East & $<5,<6,<7$ & 18 \\
\hline 24 & Luo J. $(2013, \mathrm{E})$ & $\mathrm{C}$ & NR & 1086 & 72.2 & 8.3 & Urban & 57.27 & Shanghai & East & $\begin{array}{l}\text { M, SD, }<6,<7,>8, \\
\text { SL, BT }\end{array}$ & 20 \\
\hline 25 & $\begin{array}{l}\text { Zhu Y.J. et al. (2013, } \\
\text { C) }\end{array}$ & M, S, R, C & 2012 & 4115 & 65.89 & 4.95 & Mixed & 51.01 & Jilin & Middle & $<6$ & 18 \\
\hline 26 & Yue J. et al. $(2013$, C) & $\mathrm{S}$ & 2011-2012 & 758 & 72.25 & 8.25 & NR & 43.93 & Xi'an/Shannxi & West & $<6,>8$ & 18 \\
\hline 27 & $\begin{array}{l}\text { Liu J.F. et al. (2014, } \\
\text { C) }\end{array}$ & M, R, S & 2012-2013 & 5470 & 74.56 & 6.73 & Urban & 47.04 & Changsha/Hunan & Middle & $\mathrm{M}, \mathrm{SD}$ & 20 \\
\hline 28 & $\begin{array}{l}\text { Zhang Y. et al. (2014, } \\
\text { C) }\end{array}$ & C & 2012 & 1500 & 70.24 & 8.44 & Urban & 49.33 & Tangshan/Hebei & East & $<5,<6,<7$ & 19 \\
\hline 29 & $\begin{array}{l}\text { Zhao J.X. et al. (2014, } \\
\text { C) }\end{array}$ & $\mathrm{R}$ & 2010 & 358 & NR & NR & Urban & 45.81 & Guangzhou/Guangdong & East & $>8$ & 18 \\
\hline 30 & Yu S. et al. $(2015, \mathrm{E})$ & $\mathrm{M}, \mathrm{S}, \mathrm{R}, \mathrm{C}$ & 2012-2013 & 1717 & 71.18 & 4.97 & Rural & 49.45 & Liaoning & Middle & $\mathrm{M}, \mathrm{SD}$ & 20 \\
\hline 31 & $\begin{array}{l}\text { Wang X.J.et al. } \\
(2015, \text { C) }\end{array}$ & $S, R$ & NR & 526 & 70.24 & NR & Rural & 52.28 & $\begin{array}{l}\text { Shan county, Cao } \\
\text { county/Shandong }\end{array}$ & East & $<6$ & 17 \\
\hline 32 & $\begin{array}{l}\text { Wang K.Y. et al. } \\
(2015, \text { C) }\end{array}$ & S, C, R & 2013 & 4002 & 73.34 & 6.01 & Rural & 51.42 & Yichang/Hubei & Middle & $\mathrm{M}, \mathrm{SD},<7,>9$ & 20 \\
\hline 33 & Liu H. et al. $(2016, \mathrm{E})$ & M, S, PPS & 2011-2012 & 5616 & NR & NR & NR & 47.19 & 28 provinces & & $\mathrm{M}, \mathrm{SD},<6,<7,>8$ & 20 \\
\hline 34 & $\begin{array}{l}\text { Zhi T.F. et al. (2016, } \\
\text { E) }\end{array}$ & $\mathrm{S}, \mathrm{R}$ & 2014 & 1756 & 75.3 & 3.9 & Rural & 53.30 & Rugao/Jiangsu & East & $<6,>8$ & 19 \\
\hline 35 & $\begin{array}{l}\text { Lv Q.J. et al. (2016, } \\
\text { C) }\end{array}$ & C, R & NR & 820 & 70.7 & 5.9 & Urban & 52.32 & Pingdingshan/Henan & Middle & $<5,>9$ & 21 \\
\hline 36 & $\begin{array}{l}\text { Qi S.G. et al. }(2016 \text {, } \\
\text { C) }\end{array}$ & $\mathrm{M}, \mathrm{S}, \mathrm{C}, \mathrm{R}$ & 2013 & 51774 & NR & NR & Mixed & 54.81 & $\begin{array}{l}\text { 31provinces, Xinjiang } \\
\text { Production and Construction } \\
\text { Corps }\end{array}$ & & $\mathrm{M}, \mathrm{SD},<7$ & 19 \\
\hline 37 & $\begin{array}{l}\text { Zhong X.X. et al. } \\
(2016, C)\end{array}$ & S, C, R & 2014 & 196 & 70.2 & 7.3 & Rural & 52.55 & Ma'anshan/Anhui & Middle & M, SD & 17 \\
\hline
\end{tabular}

NR=Not Reported; $\mathrm{SD}=$ Standard deviation; C; Chinese, E: English.

Sleep Information: $\mathrm{M}=$ mean of Sleep duration; $\mathrm{SD}=$ standard deviation; $<5$ refers to the percentage of participants with a sleep duration $<5$ hours/day, $<6$ refers to that $<6$ hours/day, $<7$ refers to that $<7$ hours/day,

$>8$ refers to that $>8$ hours $/$ day and $>9$ refers to that $>9$ hours $/$ day; $\mathrm{TB}=$ time to go to bed, $\mathrm{SL}=$ sleep latency, TG=time to get up, $\mathrm{BT}=$ bed time.

Sampling Method: $C=$ Cluster sampling; $M=$ Multistage sampling; $R=$ Random sampling; $S=$ Stratified sampling; $P P S=$ sampling with probability proportional to size. 
Table 2. Proportion of sleep duration with different cut-off values

\begin{tabular}{|c|c|c|c|c|c|c|c|c|c|}
\hline \multirow{2}{*}{$\begin{array}{l}\text { Cut-off of sleep duration } \\
\text { (Number of studies) }\end{array}$} & \multirow[t]{2}{*}{ Proportion (\%) } & \multicolumn{2}{|c|}{$95 \% \mathrm{CI}(\%)$} & \multirow[t]{2}{*}{ Events } & \multirow{2}{*}{$\begin{array}{l}\text { Sample } \\
\text { size }\end{array}$} & \multirow[t]{2}{*}{$I^{2}(\%)$} & \multirow[t]{2}{*}{$P$} & \multicolumn{2}{|c|}{ Publication bias } \\
\hline & & & & & & & & $P$ (Egger's Test) & $P$ (Begg's Test) \\
\hline$<5$ hours/day (5) & 18.8 & 1.7 & 35.9 & 823 & 4213 & 99.7 & $<0.001$ & 0.150 & 0.462 \\
\hline$<6$ hours/day (15) & 26.7 & 19.7 & 33.7 & 16742 & 55074 & 99.7 & $<0.001$ & 0.821 & 0.553 \\
\hline$<7$ hours/day (11) & 42.3 & 34.8 & 49.8 & 28118 & 85729 & 99.8 & $<0.001$ & 0.219 & 0.436 \\
\hline$>8$ hours/day (10) & 22.6 & 13.9 & 31.4 & 8660 & 40040 & 99.7 & $<0.001$ & 0.604 & 0.474 \\
\hline$>9$ hours/day (9) & 17.6 & 12.4 & 22.9 & 6260 & 37297 & 99.3 & $<0.001$ & 0.221 & 1.000 \\
\hline
\end{tabular}

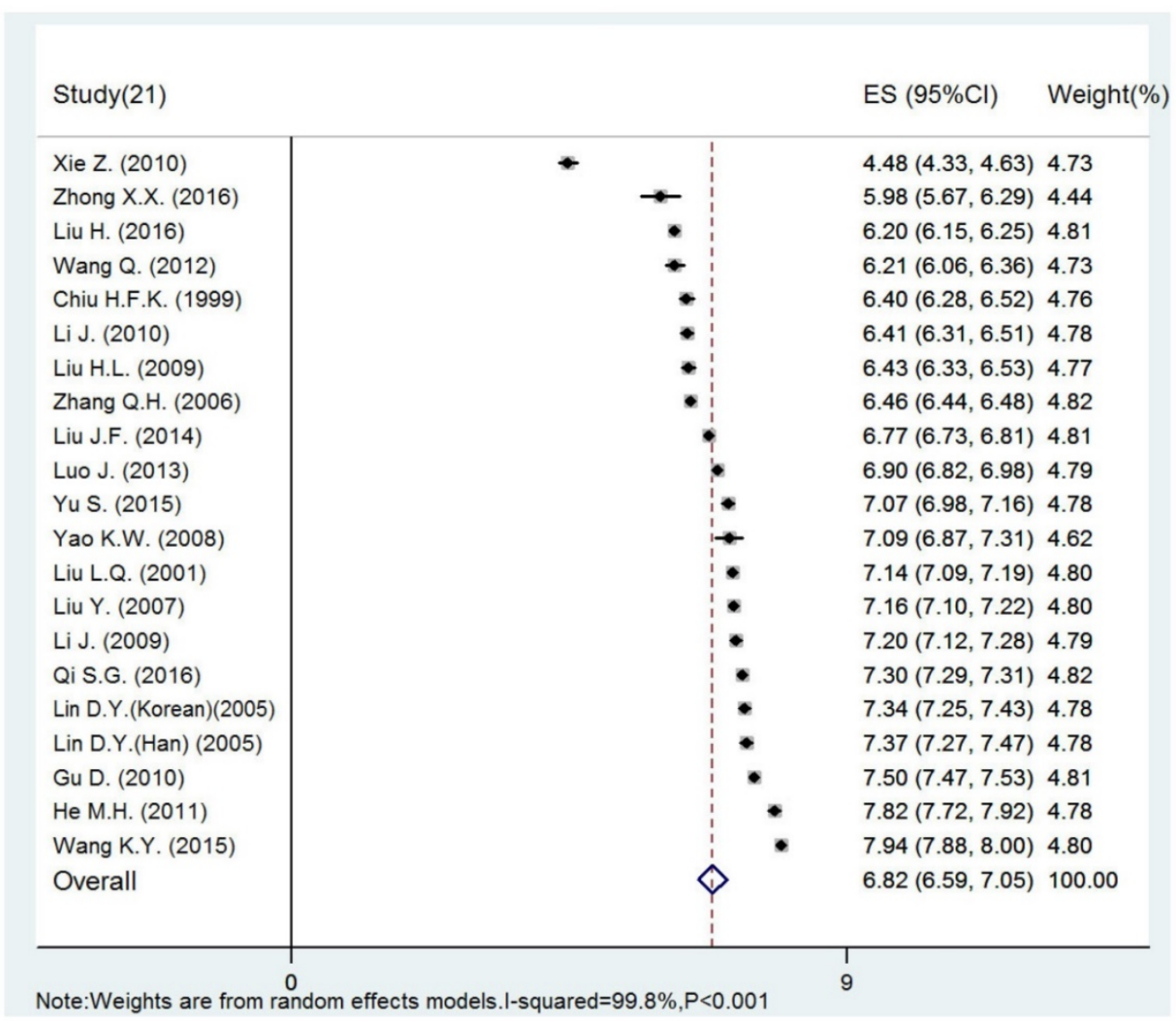

Figure 2. Forest plot of the mean sleep duration

The results of subgroup analyses using mean sleep duration, the proportion of long ( $>9$ hours/day) and short sleep ( $<6$ hours/day) are shown in Table 3. The results using other cut-offs for sleep duration are shown in Table S2. The proportion of short sleepers ( $<6$ hours/day) was $38.3 \%$ (95\% CI: $20.7 \%-59.6 \%$ ) in rural areas, while it was $17.0 \%$ (95\% CI: $8.6 \%-30.8 \%$ ) in urban areas, but the difference did not reach a significance level. Meta-regression analyses revealed that sample size and survey time did not have significant impact on the results $(P>0.05)$.

\section{Sleep habits}

The pooled time to go to bed of 8 studies with 33,517 subjects was 09:03 pm (95\% CI: 08:48 pm-09:18 $\mathrm{pm})$. The pooled mean sleep latency of 6 studies with 31,107 subjects was 30.66 minutes (95\% CI: 20.32 minutes - 41.00 minutes). The mean time to get up of 7 studies with 31,213 subjects was 05:24 am (95\% CI: 04:58 am - 05:50 am) and the mean bedtime was 7.82 hours (95\% CI: 7.43 hours - 8.21 hours) (Table S1).

The time to go to bed for older adults in the east area was later than in the middle area (09:23 pm, 95\% CI: 09:06 pm - 09:41 pm vs. 08:43 pm, 95\% CI: 08:27 pm - 09:00 pm), but the former group had longer bedtime than the latter group ( 8.10 hours, $95 \%$ CI: 7.87 hours 8.33 hours vs. 7.41 hours, $95 \%$ CI: 7.13 hours -7.70 hours). Subgroup analyses of sleep habits are shown in the Table S3.

\section{Sensitivity analyses}

After removing each study sequentially, the results from remained studies were still consistent with the primary results. 
Table 3. Subgroup analyses of sleep duration

\begin{tabular}{|c|c|c|c|c|c|c|c|c|c|}
\hline \multicolumn{10}{|c|}{ A. Subgroup analyses of night sleep duration } \\
\hline \multirow{4}{*}{$\begin{array}{l}\text { Subgroups } \\
\text { Age groups } \\
\text { (years) }\end{array}$} & $\begin{array}{l}\text { Categories } \\
\text { (Number of studies) }\end{array}$ & Mean & SE & \multicolumn{2}{|l|}{$95 \% \mathrm{CI}$} & Sample size & $I^{2}(\%)$ & $P$ & $Q(P)$ \\
\hline & $60 \sim(4)$ & 6.68 & 0.41 & 5.88 & 7.48 & 2748 & 99.4 & $<0.001$ & $0.33(0.85)$ \\
\hline & $70 \sim(4)$ & 6.53 & 0.41 & 5.73 & 7.33 & 2957 & 99.5 & $<0.001$ & \\
\hline & $\geq 80$ (4) & 6.86 & 0.41 & 6.05 & 7.67 & 1504 & 98.9 & $<0.001$ & \\
\hline \multirow[t]{2}{*}{ Gender } & Female (11) & 6.52 & 0.18 & 6.12 & 6.86 & 6666 & 99.0 & $<0.001$ & $1.33(0.25)$ \\
\hline & Male (11) & 6.81 & 0.18 & 6.46 & 7.15 & 5823 & 98.3 & $<0.001$ & \\
\hline \multirow[t]{2}{*}{ Region } & Rural (6) & 6.63 & 0.22 & 6.19 & 7.07 & 9835 & 99.8 & $<0.001$ & $0.59(0.44)$ \\
\hline & Urban (11) & 6.84 & 0.17 & 6.52 & 7.17 & 38978 & 99.4 & $<0.001$ & \\
\hline \multirow[t]{2}{*}{ Sample size } & $\leq 1500(11)$ & 6.66 & 0.17 & 6.33 & 6.99 & 7780 & 99.5 & $<0.001$ & $1.90(0.17)$ \\
\hline & $>1500(10)$ & 7.00 & 0.17 & 6.65 & 7.34 & 115067 & 99.9 & $<0.001$ & \\
\hline \multirow[t]{3}{*}{ Area } & East (11) & 6.94 & 0.20 & 6.55 & 7.33 & 50502 & 99.8 & $<0.001$ & $1.31(0.52)$ \\
\hline & Middle (8) & 6.77 & 0.22 & 6.33 & 7.21 & 14551 & 99.7 & $<0.001$ & \\
\hline & West (1) & 6.21 & 0.08 & 6.06 & 6.36 & 404 & - & - & \\
\hline \multirow[t]{2}{*}{ Survey time } & 1999-2010 (10) & 6.75 & 0.19 & 6.38 & 7.12 & 49515 & 99.8 & $<0.001$ & $0.16(0.69)$ \\
\hline & 2011-2016 (9) & 6.86 & 0.20 & 6.47 & 7.26 & 72059 & 99.8 & $<0.001$ & \\
\hline \multirow{2}{*}{$\begin{array}{l}\text { Language of } \\
\text { publications }\end{array}$} & Chinese (15) & 6.82 & 0.15 & 6.52 & 7.10 & 97569 & 99.8 & $<0.001$ & $0.04(0.85)$ \\
\hline & English (6) & 6.86 & 0.24 & 6.40 & 7.32 & 25278 & 99.7 & $<0.001$ & \\
\hline \multicolumn{10}{|c|}{ B. Subgroup analyses of the rate of short sleep duration ( $<6$ hours/day) } \\
\hline Subgroups & $\begin{array}{l}\text { Categories } \\
\text { (Number of studies) }\end{array}$ & $\begin{array}{l}\text { Proportion } \\
(\%)\end{array}$ & $95 \%$ & & Events & Sample size & $I^{2}(\%)$ & $P$ & $Q(P)$ \\
\hline \multirow[t]{2}{*}{ Region } & Rural (4) & 38.3 & 20.7 & 59.6 & 1792 & 3634 & 99.2 & $<0.001$ & $3.48(0.06)$ \\
\hline & Urban (5) & 17.0 & 8.6 & 30.8 & 9176 & 28545 & 99.2 & $<0.001$ & \\
\hline \multirow[t]{2}{*}{ Sample size } & $\leq 1700(8)$ & 21.0 & 14.8 & 28.9 & 1517 & 6120 & 99.4 & $<0.001$ & $1.36(0.24)$ \\
\hline & $>1700(8)$ & 27.7 & 19.7 & 37.5 & 15225 & 48954 & 99.6 & $<0.001$ & \\
\hline \multirow[t]{3}{*}{ Area } & East (9) & 19.0 & 12.4 & 27.9 & 11335 & 37843 & 99.5 & $<0.001$ & $3.89(0.14)$ \\
\hline & Middle (4) & 35.9 & 20.9 & 54.3 & 3330 & 10857 & 99.7 & $<0.001$ & \\
\hline & West (2) & 30.0 & 12.9 & 11.9 & 835 & 2416 & 98.8 & $<0.001$ & \\
\hline \multirow[t]{2}{*}{ Survey time } & $1997-2012(5)$ & 29.8 & 18.8 & 43.9 & 10762 & 33870 & 99.7 & $<0.001$ & $0.16(0.69)$ \\
\hline & $2013-2016(5)$ & 26.3 & 16.2 & 39.6 & 4557 & 13745 & 99.4 & $<0.001$ & \\
\hline \multirow{2}{*}{$\begin{array}{l}\text { Language of } \\
\text { publications }\end{array}$} & Chinese (12) & 24.9 & 17.0 & 32.8 & 13740 & 46616 & 99.5 & $<0.001$ & $1.30(0.25)$ \\
\hline & English (3) & 33.8 & 15.3 & 52.3 & 3002 & 8458 & 99.5 & $<0.001$ & \\
\hline C. Subgroup & yses of the rate of lon & duration ( $>$ & ours/c & & & & & & \\
\hline Subgroups & $\begin{array}{l}\text { Categories } \\
\text { (Number of studies) }\end{array}$ & $\begin{array}{l}\text { Proportion } \\
(\%)\end{array}$ & $95 \%$ & & Events & Sample size & $I^{2}(\%)$ & $P$ & $Q(P)$ \\
\hline Region & Rural (4) & 18.7 & 13.5 & 25.2 & 3745 & 14013 & 98.6 & $<0.001$ & $0.94(0.33)$ \\
\hline & Urban (2) & 24.1 & 15.7 & 35.2 & 2059 & 7799 & 91.7 & $<0.001$ & \\
\hline Sample size & $\leq 3100(5)$ & 17.9 & 11.7 & 26.4 & 1805 & 8030 & 98.8 & $<0.001$ & $0.51(0.48)$ \\
\hline & $>3100(4)$ & 14.3 & 8.8 & 22.4 & 4455 & 29267 & 99.5 & $<0.001$ & \\
\hline Area & East (4) & 23.3 & 14.2 & 35.8 & 4013 & 16182 & 99.3 & $<0.001$ & $1.64(0.44)$ \\
\hline & Middle (6) & 15.8 & 10.2 & 23.6 & 3022 & 16224 & 99.5 & $<0.001$ & \\
\hline & West (1) & 23.3 & 21.7 & 25.0 & 582 & 2497 & - & - & \\
\hline Survey time & 1997-2006 (3) & 21.0 & 12.5 & 33.0 & 3917 & 21496 & 99.6 & $<0.001$ & $0.66(0.42)$ \\
\hline & $2007-2013(3)$ & 15.6 & 9.0 & 25.6 & 1798 & 9279 & 98.9 & $<0.001$ & \\
\hline Language of & Chinese (8) & 15.7 & 11.0 & 20.4 & 5244 & 34218 & 99.0 & $<0.001$ & $4.36(0.04)$ \\
\hline publications & English (1) & 33.0 & 31.3 & 34.7 & 1016 & 3079 & - & - & \\
\hline
\end{tabular}

\section{Discussion}

In this meta-analysis, the pooled data of 21 studies showed that the mean sleep duration in Chinese older adults was 6.82 hours/day. In this group, 26.7\% reported sleeping less than 6 hours and $17.6 \%$ sleeping more than 9 hours per day. On average, they went to bed at 09:03 pm and got up at 05:24 am. Their mean time spent in bed was 7.82 hours during which they needed 30.66 minutes to fall asleep. In terms of subgroup analysis, a greater proportion of older adults in rural areas had short sleep duration ( $<6$ hours/day) than their urban counterparts.

The mean sleep duration in this meta-analysis (6.82 hours/day) is shorter than the duration (7.5 hours/day) from another large survey of 15,638 older Chinese adults in 22 provinces [13], but is similar to that found in Europe (6.95 hours/day) [24]. A multi-ethnic study on sleep duration showed that the proportions of short sleepers in white, black, Hispanic and Chinese people were $19.3 \%, 43.4 \%, 31.5 \%$ and $37.1 \%$, respectively [25]. Apart from the use of different definitions of short sleep, other factors, such 
as living rhythm, lifestyle, sleeping environment, chronic medical conditions, psychiatric disorders and outdoor activities, are significantly associated with sleep duration in older adults [24, 26].

China has been experiencing rapid urbanization and economic growth in the past decades, which may have a negative impact on the health of older adults [27]. For example, increased consumption of stimulant drinks, such as tea, coffee and energy drinks, increasing nightlife and widespread use of electronic devices often interfere with 'circadian rhythm' including sleep pattern. However, the survey year of the included studies did not show a significant moderating effect on sleep duration in subgroup analysis.

There is compelling evidence that short sleep duration could increase the risk of obesity, coronary heart disease, all-cause mortality, and was an important risk of non-successful aging $[1,5,28,29]$. In contrast, long sleep duration was positively associated with cardiovascular diseases, stroke and mortality [1, 2, 4, 28]. Moreover, cognition and memory impairment were common in both short and long sleepers [3, 6, 30-32].

Previous studies found that low economic status is associated with greater sleep disturbance and short or long sleep duration [33, 34]. Considering that economic status is higher in the eastern region and urban areas than in the central region and rural areas in China, subgroup analyses were conducted between different areas defined by the Chinese economic zone. We found the proportion of short sleepers $(<6$ hours/day) in rural area (38.3\%) was higher than in urban area $(17.0 \%)$; the proportion in central region $(35.9 \%)$ was higher than in the eastern region of China $(19.0 \%)$, which support the association between economic status and sleep pattern. In addition, we found that Chinese older adults usually go to sleep almost an hour earlier than their counterparts in the USA, while the sleep latency of the Chinese old people were similar to those in the USA and Europe $[24,35]$.

The results should be interpreted with caution due to several limitations. First, inconsistent criteria were used in assessing the sleep duration across studies [36]. Second, sleep data were self-reported which may lead to recall bias. Third, heterogeneity remained in the subgroup analyses, since heterogeneity cannot be avoided in large meta-analysis of epidemiological surveys [37-39].

In conclusion, short sleep duration is common in Chinese older adults. Given its adverse effects, effective measures should be implemented to improve the sleep patterns in this population.

\section{Supplementary Material}

Supplementary figures and tables. http:/ / www.ijbs.com/v13p0682s1.pdf

\section{Acknowledgements}

The study was supported by the University of Macau (SRG2014-00019-FHS; MYRG2015-00230-FHS; MYRG2016-00005-FHS). The University of Macau had no role in the study design, generating or interpreting the results and publication of the study.

\section{Competing Interests}

The authors have declared that no competing interest exists.

\section{References}

1. Itani O, Jike M, Watanabe N, Kaneita Y. Short sleep duration and health outcomes: a systematic review, meta-analysis, and meta-regression. Sleep Med. 2017; 32: 246-56.

2. Stenholm S, Kronholm E, Sainio P, Borodulin K, Era P, Fogelholm M, et al. Sleep-related factors and mobility in older men and women. J Gerontol A Biol Sci Med Sci. 2010; 65: 649-57.

3. Xu L, Jiang CQ, Lam TH, Liu B, Jin YL, Zhu T, et al. Short or long sleep duration is associated with memory impairment in older Chinese: the Guangzhou Biobank Cohort Study. Sleep. 2011; 34: 575-80.

4. Song Q, Liu X, Zhou W, Wang L, Zheng X, Wang X, et al. Long Sleep Duration and Risk of Ischemic Stroke and Hemorrhagic Stroke: the Kailuan Prospective Study. Scientific reports. 2016; 6: 33664.

5. Liu H, Byles JE, Xu X, Zhang M, Wu X, Hall JJ. Association between nighttime sleep and successful aging among older Chinese people. Sleep Med. 2016; 22: $18-24$.

6. Lo JC, Loh KK, Zheng H, Sim SK, Chee MW. Sleep duration and age-related changes in brain structure and cognitive performance. Sleep. 2014; 37: 1171-8.

7. Ferrie JE, Shipley MJ, Cappuccio FP, Brunner E, Miller MA, Kumari M, et al. A prospective study of change in sleep duration: associations with mortality in the Whitehall II cohort. Sleep. 2007; 30: 1659-66.

8. Heslop P, Smith GD, Metcalfe C, Macleod J, Hart C. Sleep duration and mortality: The effect of short or long sleep duration on cardiovascular and all-cause mortality in working men and women. Sleep medicine. 2002; 3: 305-14.

9. Kinsella KG, Phillips DR. Global aging: The challenge of success. Population Reference Bureau Washington. 2005; 60

10. Chatterji S, Kowal P, Mathers C, Naidoo N, Verdes E, Smith JP, et al. The health of aging populations in China and India. Health affairs (Project Hope). 2008; 27: 1052-63.

11. Ministry of Civil Affairs of the People's Republic of China. Statistical Communique on Social Services Development. 2015.

12. St-Onge MP, Grandner MA, Brown D, Conroy MB, Jean-Louis G, Coons M, et al. Sleep Duration and Quality: Impact on Lifestyle Behaviors and Cardiometabolic Health: A Scientific Statement From the American Heart Association. Circulation. 2016; 134: e367-e86.

13. Gu D, Sautter J, Pipkin R, Zeng Y. Sociodemographic and health correlates of sleep quality and duration among very old Chinese. Sleep. 2010; 33: 601-10.

14. Qi SG, Wang ZH, Li ZX, Zhang M, Huang ZJ, Deng Q, et al. Sleep duration among elderly in 2013: association with stroke. Chinese Journal of Medical Frontier(electronic edition). 2016; 8: 109-14.

15. Liu H, Byles JE, Xu X, Zhang M, Wu X, Hall JJ. Association between nighttime sleep and successful aging among older Chinese people. Sleep Medicine. 2016; 22: $18-24$.

16. Hirshkowitz M, Whiton K, Albert SM, Alessi C, Bruni O, DonCarlos L, et al. National Sleep Foundation's sleep time duration recommendations: methodology and results summary. Sleep Health. 2015; 1: 40-3.

17. von Elm E, Altman DG, Egger M, Pocock SJ, Gotzsche PC, Vandenbroucke JP. The Strengthening the Reporting of Observational Studies in Epidemiology (STROBE) statement: guidelines for reporting observational studies. PLoS medicine. 2007; 4: e296.

18. Cao XL, Zhong BL, Xiang YT, Ungvari GS, Lai KY, Chiu HF, et al. Prevalence of suicidal ideation and suicide attempts in the general population of China: A meta-analysis. Int J Psychiatry Med. 2015; 49: 296-08.

19. Higgins JP, Thompson SG, Deeks JJ, Altman DG. Measuring inconsistency in meta-analyses. BMJ (Clinical research ed). 2003; 327: 557-60.

20. Higgins JP, Green S. Cochrane handbook for systematic reviews of interventions. John Wiley \& Sons; 2011.

21. Begg CB, Mazumdar M. Operating characteristics of a rank correlation test for publication bias. Biometrics. 1994; 50: 1088-101. 
22. Egger M, Davey Smith G, Schneider M, Minder C. Bias in meta-analysis detected by a simple, graphical test. BMJ (Clinical research ed). 1997; 315: 629-34.

23. Lin DY, Han CJ, Shen HM, Ping LYY. Analysis to status and influential factors of sleep quality of the elderly in community of Yanji city. Journal of Yanbian University(Medical Sciecnces). 2005: 273-5.

24. Ohayon MM. Interactions between sleep normative data and sociocultural characteristics in the elderly. J Psychosom Res. 2004; 56: 479-86.

25. Chen X, Wang R, Zee P, Lutsey PL, Javaheri S, Alcantara C, et al. Racial/Ethnic Differences in Sleep Disturbances: The Multi-Ethnic Study of Atherosclerosis (MESA). Sleep. 2015; 38: 877-88.

26. Xiang YT, Wong TS, Tsoh J, Ungvari GS, Correll CU, Ko FW, et al. Insomnia in older adults with chronic obstructive pulmonary disease (COPD) in Hong Kong: a case-control study. Copd. 2014; 11: 319-24.

27. Zhang T, Tan H, Wu Y, Han B, Wang T. Urban Older Adults Becoming Unhealthier in Modern China: A Cross-Temporal Meta-Analysis. Psychological reports. 2016; 118: 737-47.

28. Da Silva AA, De Mello RG, Schaan CW, Fuchs FD, Redline S, Fuchs SC. Sleep duration and mortality in the elderly: a systematic review with meta-analysis. BMJ Open. 2016; 6: e008119.

29. Zhi TF, Sun XM, Li SJ, Wang QS, Cai J, Li LZ, et al. Associations of sleep duration and sleep quality with life satisfaction in elderly Chinese: The mediating role of depression. Archives of Gerontology and Geriatrics. 2016; 65: $211-7$

30. Devore EE, Grodstein F, Schernhammer ES. Sleep Duration in Relation to Cognitive Function among Older Adults: A Systematic Review of Observational Studies. Neuroepidemiology. 2016; 46: 57-78.

31. Ferrie JE, Shipley MJ, Akbaraly TN, Marmot MG, Kivimaki M, Singh-Manoux A. Change in sleep duration and cognitive function: findings from the Whitehall II Study. Sleep. 2011; 34: 565-73.

32. Virta JJ, Heikkila K, Perola M, Koskenvuo M, Raiha I, Rinne JO, et al Midlife sleep characteristics associated with late life cognitive function. Sleep. 2013; 36: 1533-41, 41a.

33. Grandner MA, Patel NP, Gehrman PR, Xie D, Sha D, Weaver T, et al. Who gets the best sleep? Ethnic and socioeconomic factors related to sleep complaints. Sleep Med. 2010; 11: 470-8.

34. Wang KY, Zhang XH, Yi LP, Huang WD, Sun B, Zhou ZH. Self-rated sleep status and related factors among people aged 65 years and over in Yiling rural mountainous area, Yichang. South China journal of Preventive Medicine. 2015; 41: 419-23.

35. Habte-Gabr E, Wallace RB, Colsher PL, Hulbert JR, White LR, Smith IM. Sleep patterns in rural elders: demographic, health, and psychobehavioral correlates. J Clin Epidemiol. 1991; 44: 5-13.

36. Xiang YT, Ma X, Lu JY, Cai ZJ, Li SR, Xiang YQ, et al. Relationships of sleep duration with sleep disturbances, basic socio-demographic factors, and BMI in Chinese people. Sleep Med. 2009; 10: 1085-9.

37. Winsper C, Ganapathy R, Marwaha S, Large M, Birchwood M, Singh SP. A systematic review and meta-regression analysis of aggression during the first episode of psychosis. Acta Psychiatr Scand. 2013; 128: 413-21.

38. Long J, Huang G, Liang W, Liang B, Chen Q, Xie J, et al. The prevalence of schizophrenia in mainland China: evidence from epidemiological surveys. Acta Psychiatr Scand. 2014; 130: 244-56.

39. Li Y, Cao XL, Zhong BL, Ungvari GS, Chiu HF, Lai KY, et al. Smoking in male patients with schizophrenia in China: A meta-analysis. Drug Alcohol Depend. 2016; 162: 146-53. 